\title{
Laboratório de comunicação: ampliando as habilidades do estudante de medicina para a prática da entrevista
}

\author{
Mario Alfredo De Marco ${ }^{1}$ \\ Ana Luiza Vessoni² \\ Augusto Capelo ${ }^{3}$ \\ Cíntia Camargo Dias ${ }^{4}$
}

\section{Introdução}

Um obstáculo importante para o aprendizado de técnicas para a realização da entrevista médica é a ideia de que este preparo não é necessário (como se fosse inerente ao profissional já saber entrevistar) ou possível.

Hoje, essa postura é amplamente questionada e inúmeros trabalhos têm investigado os diferentes tópicos nesse campo, entre os quais:

A técnica para realizar entrevista exige aprendizado específico? É possível aprender essas técnicas ou isso depende exclusivamente de características pessoais? Esse aprendizado deve ser parte do currículo médico? Em caso afirmativo, como inserir esse aprendizado no currículo?

Acreditamos que a questão a seguir deve ser a primeira a ser abordada: $E$ possível ensinar habilidades para a realização da entrevista ou essas habilidades são características pessoais que independem de aprendizado e treinamento? A validade de todas as outras perguntas depende da resposta a esta questão básica.

Hoje, sabemos que essa resposta é afirmativa: inúmeros trabalhos focando o treinamento permitem afirmar com segurança que essas habilidades podem ser ensinadas e aprendidas.

Mais detalhadamente, esses trabalhos e pesquisas revelam que:

- a qualidade da entrevista e da relação entre profissional e paciente é amplamente dependente das habilidades do profissional para conhecer e manejar o processo de comunicação (Borrell, 2004; Wissow, Kimel, 2002; Rodríguez Salvador, 2001, Aspegren, 1999);

- a qualidade da entrevista e da comunicação favorecem adesão ao tratamento e evolução (Aspegren, 1999; Bartlett et al., 1984);

- habilidades de comunicação podem ser ensinadas e aperfeiçoadas (Hulsman et al., 1999; Henwood, Altmaier, 1996);

Essas confirmações nos remetem a outras observações e questionamentos:

- como incluir esse preparo em nossa formação profissional?

- como saber se já estou preparado?

- como alcançar preparo e aperfeiçoamento?

Há várias décadas, o campo tem sido objeto de atenção e experimentação; e um formato para inclusão desse treinamento nos programas, a técnica de vídeo-

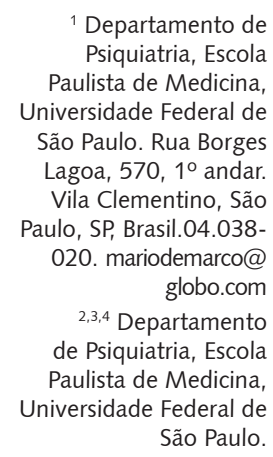


feedback interativa, vem demonstrando efetividade e superioridade em relação a outros métodos (Roter et al., 2004; Yedidia et al., 2003; Humphris, Kaney, 2000). A técnica consiste, sucintamente, na gravação de entrevistas com pacientes reais e/ou dramatizados (role-playing) e feedback interativo por meio do exame do material gravado.

Em nosso trabalho na Unifesp, por intermédio dos programas de Psicologia Médica e em integração com outros módulos interdisciplinares, temos desenvolvido ações voltadas para esse tópico.

A título de contextualização, segue breve histórico de nossa atividade: A Psicologia Médica, introduzida no currículo médico da Escola Paulista de Medicina em 1956, integrando o programa do terceiro ano do curso, está presente, atualmente, no currículo do primeiro ao quinto ano.

Diferentemente do modelo inicial, basicamente teórico (aulas para toda a turma), o trabalho atual é, primordialmente, vivencial e participativo, realizado em pequenos grupos (dez a vinte alunos), de forma a proporcionar espaço para discussões e elaboração das vivências (De Marco, 2006, 2003). Ao foco inicial, dirigido essencialmente para o estudo do desenvolvimento da personalidade, adicionou-se o estudo da relação e comunicação.

Na prática, o curso de Psicologia Médica está cada vez mais articulado à nossa perspectiva de educação continuada. Nessa perspectiva, executada integradamente, por meio de diferentes atividades (Psicologia Médica, Interconsulta, programas de Ligação em Saúde Mental, programas de capacitação e em múltiplas oportunidades de contato com estudantes e profissionais), o curso regular de Psicologia Médica, em sua parte prática (introduzida cada vez mais cedo), compõe o quadro mais amplo de um programa em que, no hospital e demais cenários de atendimento, acompanhamos e/ou estamos disponíveis para o estudante, ao longo de toda sua evolução na formação (De Marco, 2003).

Entre as várias iniciativas para diminuir a fragmentação dos programas de ensino na graduação (Garcia et al., 2007; Trindade et al., 2005; Cyrino, Rizzato, 2004), planejamos o módulo de "Semiologia Integrada", inserido no currículo do curso médico desde 2004. O módulo é parte de um conjunto mais amplo de ações voltadas para promover aproximação antecipada aos cenários da prática (Sayd et al., 2003; Santos et al., 2003). No caso da abordagem semiológica, o programa, além da aproximação antecipada, foi planejado para corrigir o seguinte quadro: o programa de psicologia médica (primeiro e segundo ano do curso médico) colocava o aluno em contato com conhecimentos e treinamento para capacitá-lo a perceber, valorizar e manejar, nas entrevistas com os pacientes, os aspectos psicossociais, a relação e a comunicação, enfatizando a importância de uma visão processual do adoecer. Paradoxalmente, nos anos posteriores, quando recebia treinamento nas clínicas específicas, o aluno se defrontava com uma visão e prática focalizada na doença e, basicamente, nos sintomas e aspectos físicos.

Para corrigir esta distorção, a proposta do módulo de Semiologia Integrada foi a de envolver, numa prática conjunta, professores das diferentes áreas e disciplinas. Assim, professores dos diferentes ciclos de vida contemplados pelo curso (Pediatria, Clínica Médica, Obstetrícia, Ginecologia e Geriatria) participam tanto do acompanhamento das entrevistas como das discussões, lado a lado com o professor de Psicologia Médica. O módulo ocorre em continuidade ao curso de Psicologia Médica, ministrado no primeiro semestre, no qual já tem lugar uma iniciação no treinamento de entrevistas. São mantidas turmas (18 a vinte alunos) e coordenador (professor de Psicologia Médica) de cada grupo.

Adicionalmente, reconhecendo a importância do desenvolvimento do ensino da comunicação nos cursos de graduação em medicina, em consonância com as novas Diretrizes Curriculares Nacionais (Rossi, Batista, 2006), ampliamos o investimento nesse campo com a atividade "laboratório de comunicação", inserida no programa de Psicologia Médica do segundo ano, antecedendo a prática das entrevistas com os pacientes. A atividade destina-se a aprofundar o conhecimento e vivência das técnicas de comunicação por meio de discussões e exercícios com apoio de gravação de imagens. A implementação do programa foi precedida de preparo realizado por meio do estudo de diferentes experiências, particularmente de profissionais e pesquisadores ligados à SemFYC (Sociedad Española de Medicina de Família y Comunitária) e à John Hopkins Bloomberg School of Public Health, bem como por intermédio da oportunidade de contar com a vinda de profissionais dessas instituições (Fernando Orozco, Ana Sobrino e Lawrence Wissow) que promoveram atividades de discussão e capacitação com nosso grupo de professores. 
A intenção principal deste trabalho é apresentar a proposta e metodologia desta atividade (laboratório de comunicação), construída com base na experiência acumulada, e inspirada pelos trabalhos e contribuições dos vários autores mencionados, particularmente aqueles ligados à SemFYC (Sociedad Española de Medicina de Família y Comunitária) e à John Hopkins Bloomberg School of Public Health.

\section{Laboratório de comunicação - Psicologia Médica}

O objetivo geral da atividade é proporcionar ao aluno conhecimento e treinamento nas habilidades de comunicação necessárias para desempenho efetivo e eficiente da entrevista e da consulta médica.

Como objetivos específicos temos:

- Sensibilizar para os diferentes aspectos da comunicação verbal e não-verbal e sua importância na tarefa médica.

- Fomentar o respeito ao estilo pessoal e facilitar o reconhecimento das habilidades e características pessoais de cada entrevistador.

- Propiciar a evolução e ampliação de recursos técnicos e habilidades para uma comunicação mais efetiva e eficiente.

- Ajudar a identificar e lidar com situações consideradas "difíceis", sistematizando observações e procedimentos para esse fim.

\section{Metodologia}

Cada turma, de aproximadamente vinte alunos, juntamente com o professor de psicologia médica que coordena o grupo, passa pelo laboratório de comunicação (três reuniões de cento e vinte minutos por grupo). É disponibilizado previamente para o aluno material referente à atividade.

A abordagem do laboratório tem como foco principal a atividade prática e a observação/discussão/ elaboração dessa experiência, visando a evolução das habilidades para a realização de entrevistas e contatos inerentes à atividade médica. A disponibilização de material audiovisual ou material teórico destina-se ao apoio a essa atividade.

A intenção é oferecer oportunidade de ensaiar formas de atuação na entrevista clínica, que favoreçam a evolução das capacidades de comunicação.

A prática é realizada por meio da técnica de role-playing e envolve participação de alunos e professores. A representação é gravada (vídeo) e apresentada na mesma reunião, permitindo observação detalhada e detida das modalidades comunicacionais, ensaio de várias possibilidades de interação e introdução de elementos técnicos que enriqueçam a comunicação verbal e não-verbal do entrevistador.

\section{Estrutura da atividade}

Três reuniões com duração de duas horas/cada, assim distribuídas:

- Primeira reunião: 1 Comunicação não verbal; 2 Recepção / apresentação na entrevista.

- Segunda reunião: Fase exploratória da entrevista.

- Terceira reunião: Fase resolutiva da entrevista.

\section{Primeira reunião}

1 Comunicação não verbal; 2 Recepção e apresentação na entrevista.

\section{Roteiro}

É realizada apresentação da atividade (aproximadamente 15 minutos) seguida de diálogo aberto - "bate-bola" - (15 minutos) sobre a importância da comunicação na tarefa médica (tópicos: Qual 
a ideia sobre a comunicação e sua importância? Quais os fatores que facilitam e dificultam? Numa consulta médica, como numa aula, existe uma tarefa comum: como alcançar consenso quanto à tarefa comum? O que pode atrapalhar alcançar consenso e cooperação? Qual o papel da comunicação nesse processo?). Na sequência, uma exposição interativa (20 minutos) aborda tópicos relacionados ao tema da reunião. Os tópicos são apresentados para discussão no formato a seguir:

1 A comunicação não verbal

A comunicação não verbal costuma ser categorizada em: paralinguagem, proxêmica e cinésica.

Paralinguagem: se refere às qualidades da emissão vocal (altura, intensidade ritmo etc.) que fornecem informações sobre o estado afetivo do emissor, bem como outras produções vocais, como o riso, o grito, o bocejo, a tosse etc., e, podem ser agrupadas nas seguintes categorias:

- qualidade da voz, que inclui a altura do tom de voz, a qualidade de articulação e o ritmo;

- qualificadores vocais, que incluem a forma como as palavras são emitidas (extensão, timbre, intensidade);

- caracterizadores vocais, que incluem certos sons bem reconhecidos, como o suspiro, o bocejo, o riso, o choro, o grito etc.;

- secreções vocais, que incluem sons que participam do fluxo da fala sem que as palavras signifiquem alguma coisa (ahn, hum, hen, aha, pausas e outras interrupções de ritmo).

Proxêmica: se refere ao uso do espaço, envolvendo as dimensões de distância, território e ordem na comunicação humana (é muito importante nos animais); é o jogo de distâncias e proximidades que se entretecem entre as pessoas e o espaço, traduzindo as formas como se colocam e movem em relação aos outros, como gerenciam e ocupam o espaço. Define a relação que os comunicantes estabelecem entre si: a distância espacial, a orientação do corpo e do rosto, a forma como se tocam ou se evitam, o modo como dispõem e se posicionam entre os objetos e os espaços.

Em nossa atividade envolve:

- a forma como nos aproximamos do paciente (contato corporal, angulação do corpo - como sinais de aceitação, rechaço e hierarquia).

- a utilização e distribuição dos espaços (decoração, barreira da mesa etc.), propiciando um trabalho em campo tenso ou campo relaxado.

Cinésica: se refere aos gestos e movimentos corporais e estende-se por cinco áreas: contato visual, gestos, expressões faciais, postura e movimentos da cabeça.

Apresenta diferenças significativas dependendo do ciclo de vida (criança, adulto e idoso) e da sociedade e cultura (a maioria dos autores considera que não há expressões universais e que qualquer expressão facial, atitude ou posição corporal tem significados diferentes nas diversas sociedades).

2 Recepção/apresentação na entrevista

Procedimentos e cuidados:

Acolhimento: a forma como acolhemos o paciente é muito importante e influencia significativamente todo o processo. Algumas qualidades importantes do entrevistador influenciam positivamente o acolhimento. São elas:

- Qualidades de superfície: calidez, respeito e cordialidade.

- Qualidades profundas: empatia, continência emocional e assertividade.

Capacidade de observação da comunicação não verbal: é importante em toda a entrevista, mas é particularmente crítica para a fase da apresentação/recepção. Ela favorece a observação imediata de aspectos do paciente e seu eventual acompanhante que poderão facilitar a condução da entrevista, bem como a observação e a possibilidade de equacionamento de uma série de interferências e ruídos:

- Aspectos do paciente e seu eventual acompanhante: a aparência, a forma de vestir, a postura corporal (aberta, fechada...), o estilo e a postura na marcha, a forma de cumprimentar (o aperto de mão, o olhar...), o timing (particularmente em relação ao acompanhante - invasivo, passivo, colaborador) são elementos que já fornecem uma série de dados e auxiliam a manter desobstruídos os canais de comunicação, facilitando uma condução mais favorável da entrevista.

- Observação e equacionamento de interferência e ruídos: a atenção aos ruídos e interferências é também bastante útil para ajudar a diagnosticar e equacionar as perturbações no contato que podem se produzir como decorrência dos mesmos. Eles envolvem uma série de características e 
eventos que participam indiretamente do encontro: o ambiente do consultório ou centro onde se realiza o atendimento, o número de pessoas na sala de espera, o tempo de espera, o nível de ruídos e interferências no ambiente, as interrupções, as chamadas telefônicas etc.

Após a exposição interativa é apresentada a proposta de atividade prática, que, nesta primeira reunião, é a de "encenar a organização de uma sala de atendimento e situações de início de consulta". É solicitada a apresentação de voluntários e as encenações são gravadas e exibidas, permitindo um exame detalhado e detido dos elementos comunicacionais, dando ensejo à percepção na prática do cumprimento ou não dos tópicos abordados, bem como a discussão e ensaio de diferentes possibilidades.

Na sequência, a título de finalização (vinte minutos), realiza-se um breve sumário dos pontos e das questões mais importantes que se apresentaram, bem como um feedback de toda a atividade.

\section{Segunda reunião}

Fase exploratória da entrevista

\section{Roteiro}

Abre-se espaço para comentários sobre a atividade realizada na semana anterior, contextualizando e estabelecendo pontes para a atividade do dia, sobre a qual é apresentado um roteiro (aproximadamente 15 minutos). Segue-se o diálogo aberto - "bate-bola" - (15 minutos) abordando aspectos relevantes da fase exploratória da entrevista (Como se inicia uma investigação? O que vai nos orientar na investigação? O que define um bom entrevistador? Quais os principais problemas que vai enfrentar?), como aquecimento para a exposição interativa (vinte minutos) que focalizará os seguintes tópicos:

Procedimentos e cuidados:

1 Delimitação do motivo da consulta: clarificar os motivos do paciente com uma pergunta aberta do tipo: "o que o traz aqui?" ou "como posso ajudá-lo?" ou mesmo "pois não?". Delimitar todas as demandas, pois isso ajuda a priorizar: indagar, sempre, "mais alguma coisa?", até se certificar de que todas as demandas foram expressas. Quando existem muitas demandas, é importante se estabelecerem prioridades.

2 Obtenção dos dados necessários para se estabelecer a natureza do problema, as crenças e as expectativas do paciente:

- Explorar que ideias tem o paciente quanto à natureza da enfermidade. Perguntas como "o que você acha que está provocando estes sintomas?" podem ser úteis.

- Explorar os sentimentos que provocam (especialmente medos). Perguntas como "em relação a este problema, tem alguma coisa que lhe preocupa?" podem ser utilizadas.

3 Incorporar à exploração o contexto familiar, social e laboral.

Principais técnicas de apoio narrativo a serem observadas com o objetivo de facilitar a verbalização do paciente:

- Baixa reatividade: o entrevistador deixa um lapso de tempo entre a intervenção do paciente e a sua. Alguns segundos (um ou dois) entre a intervenção do paciente e a nossa evitam interrupções e favorecem a livre narração do paciente.

- Silêncio funcional: ausência de comunicação verbal com o intuito de proporcionar um tempo de meditação ao paciente, ajudar sua concentração, ou atuar como catalisador de determinadas reações emocionais no curso da entrevista.

- Facilitação: conduta verbal ou não verbal para ajudar o paciente a prosseguir ou iniciar seu relato, sem indicar nem sugerir os conteúdos do mesmo. Por exemplo, gestos de assentimento com a cabeça que indicam "continue, estou ouvindo com atenção" ou sons que carreguem um significado semelhante. Ou mais explicitamente: "continue, por favor", "e que mais?" etc.

- Empatia: conduta verbal ou não verbal que expressa solidariedade emocional sem prejulgar ética ou ideologicamente. Um contato, um sorriso, "compreendo como se sente".

- Frases por repetição: repetição de palavra ou frase pronunciada pelo paciente a fim de orientar a atenção do mesmo para aquele aspecto: "Então você me dizia que foi depois daquela discussão que teve esta forte dor de cabeça que está te preocupando". 
- Assinalamento: explicitação de emoções ou condutas. Pode ser feito por intermédio da observação e nomeação do estado de ânimo do paciente: "parece que você ficou um tanto aborrecido comigo" ou: "estou observando que você foi ficando triste...".

Após a exposição interativa, é apresentada a proposta de atividade prática que, nesta segunda reunião, é a de "realizar uma entrevista com o foco na fase exploratória".

Como material de apoio, fornecemos a parte inicial de um roteiro de entrevista que apresenta o esboço de uma situação para ser manejada e expandida. O roteiro poderá, também, ser reformulado e/ou utilizado como modelo para a construção de outros roteiros:

Médico - Dona Júlia

Paciente - Sou eu doutor.

M - Bom dia, dona Júlia, vamos entrar (cumprimenta)

Entram no consultório e sentam-se

M - Então, dona Júlia, como posso ajudá-la?

$\mathrm{P}$ - Doutor, é uma dor muito forte aqui na cabeça (coloca a mão direita na região frontal)

$M$ - Uma dor forte na cabeça... me conta mais dessa dor.

$\mathrm{P}$ - Bom doutor, é uma dor que eu já levanto com ela (tom bastante queixoso). Às vezes melhora um pouco durante o dia, à noitinha sempre está um pouco melhor...

$M$ - E faz quanto tempo que começou essa dor?

$P$ - Olha doutor, faz bem uns quinze dias...

$M$ - E a senhora já teve essa dor antes?

$P$ - Bem doutor, de vez em quando, isso me ataca, mas nunca foi tão forte como desta vez...e não passa com nada...das outras vezes eu tomava aspirina e a dor ia embora..., agora, não adianta nada a aspirina (abaixa levemente a cabeça e leva a mão à região frontal fazendo expressão de dor).

$M$ - Vejo que é uma dor muito incômoda.

P - Ah doutor, é um sofrimento (a expressão de dor persiste mas agora suavizada, o rosto se descontrai e surge um brilho no olhar que agora dirige ao médico)

$M$ - E além da dor de cabeça, mais alguma coisa?

$P$ - Não doutor, não que eu me lembre agora.

M - O que a senhora acha que pode estar causando essa dor de cabeça?

$P$ - Não sei doutor, meu medo é que possa ser um tumor. Eu queria fazer uma chapa p'ra ver o que tem dentro da cabeça.

M - E a senhora lembra de alguma coisa que aconteceu quando começou essa dor de cabeça?

$P$ - Não doutor, não aconteceu nada que eu lembre.

$M$ - E a família, como vai?

P - Ah tudo bem, doutor, meu marido é um homem muito bom, trabalhador (abaixa a cabeça, leva a mão à fronte e contrai fortemente a expressão)

M - Mas, não tem alguma coisa da família preocupando a senhora?

P - (abaixa um pouco mais a cabeça e vai emitindo um som de choro abafado).

M - (permanece um tempo em silêncio e inclinando-se na direção da paciente fala em tom acolhedor) Está muito difícil, não é?

P - (a paciente libera o choro e gradualmente vai contando sua aflição) É, doutor, minha filha de 14 anos, imagina o senhor que faz uns 15 dias me apareceu grávida...

A gravação, a discussão e a finalização seguem a mesma dinâmica já descrita na primeira atividade.

\section{Terceira reunião}

Fase resolutiva da entrevista

\section{Roteiro}

A dinâmica inicial é a mesma da segunda reunião, com diferença do tema, aqui focado na fase 
resolutiva da entrevista, a respeito do qual são propostas as seguintes questões no "bate-bola": Quais os principais cuidados no fornecimento de informações? Como se verifica a assimilação das informações? É importante/necessário negociar um consenso com o paciente sobre os procedimentos e o tratamento? O que facilita alcançar um consenso? E quando não é possível, o que fazer?

Na sequência, na exposição interativa, são abordados os seguintes tópicos:

A fase resolutiva é subdividida em 2 etapas: informativa e negociadora.

1 Etapa informativa: envolve fornecimento de informações ao paciente da maneira mais apropriada, tendo em vista:

- Responder às expectativas do paciente que deseja ser informado sobre sua saúde.

- Melhorar a adesão ao tratamento.

Há alguns erros mais importantes a evitar nesta etapa:

- Muitos conceitos por unidade de tempo.

- Uso de linguagem excessivamente técnica.

- Misturar diferentes problemas sem dar explicação concreta para cada um.

- Interromper o paciente quando este vai perguntar ou falar.

Há fatores que favorecem a boa compreensão e assimilação. Entre eles:

- Fornecer informações compreensíveis e despertar interesse.

- Detectar resistências, entender preocupações e fornecer esclarecimentos necessários.

- Comprovar a assimilação, isto é, assegurar-se de que o paciente compreendeu.

2 Etapa negociadora: envolve pactuação das condutas e orientações entre o profissional e o paciente. Implica, evidentemente, que o profissional tenha clareza quanto ao direito do paciente de participar e opinar sobre seu processo. Quando as orientações indicadas pelo profissional são colocadas em dúvida pelo paciente, estamos frente a uma negociação propriamente dita, que envolve, como passos decisivos:

- Reconhecer o direito do paciente a participar e opinar sobre seu processo.

- Explorar as crenças do paciente, mesmo quando suas opiniões foram expressas de forma agressiva ou receosa.

- Discutir as crenças mostrando incoerências, indicando contradições ou contrapondo informação. Ex: "Mudar os hábitos não é o problema, o que é um problema é não ter a pressão controlada. A pressão alta faz com que o coração precise fazer mais força para bombear o sangue".

- Tentar desenvolver técnicas de negociação e persuasão, inclusive em situações em que as opiniões do paciente nos pareçam pouco justificadas.

- Respeitar as últimas decisões do paciente.

A gravação, a discussão e a finalização seguem a mesma dinâmica descrita na segunda atividade. A diferença é que, nesta prática, além do roteiro de entrevista, são fornecidos ao aluno dados sobre a paciente, resultados dos exames e informações sobre a patologia. É uma consulta de retorno na qual a paciente deverá receber orientações de mudança de estilo de vida, tendo em vista o controle da pressão arterial e do colesterol.

Aqui, também, o roteiro é aberto, colocando um impasse para ser manejado pelo entrevistador. O roteiro deverá ser desenvolvido e expandido e/ou servir como modelo para a construção de outros roteiros:

Médico - Olá, dona Maria, como vai?

Paciente - Graças a Deus, tudo bem, doutor.

M - Então, dona Maria, estou aqui com os resultados dos seus exames.

P - Tá tudo bem, não é, doutor? Eu não sinto nada, acho que tenho uma boa saúde.

$M$ - Bem, dona Maria, na verdade os exames estão um pouco alterados...

$P$ - Nossa, doutor, é mesmo?

M - Sim, dona Maria, veja, não é nada muito grave no momento, mas é importante cuidar para

prevenir problemas no futuro

$P$ - Mas o que é que eu tenho afinal doutor?

M - Bem, Dona Maria, a senhora está com a pressão alta e o açúcar e a gordura no sangue 
também estão altos. Na verdade, sua gordura no sangue, quer dizer, seu colesterol, está desequilibrado: o colesterol "bom" está baixo e o colesterol "ruim" está alto.

$P$ - Doutor, mas eu quase não como gordura.

M - Bem, dona Maria, na verdade não é só comer gordura que faz aumentar a gordura no sangue, tem outros alimentos e, também, os hábitos, a herança...

$P$ - Bom, doutor, mas o que o senhor quer que eu faça?

M - São várias coisas, dona Maria. Precisa mudar alguns hábitos da sua vida: precisa começar a fazer exercícios, precisa diminuir bastante o sal da comida e mudar o tipo de alimentos - depois eu passo isso tudo direitinho para a senhora. Precisa também parar de tomar bebidas alcoólicas. Ah, e o cigarro também precisa parar...

P - Tudo isso, doutor? Ah não, doutor, é muita coisa. Além disso, eu estou bem. Não estou sentindo nada...

\section{Recursos humanos e materiais}

Três professores, de forma a permitir participação ativa tanto nas discussões quanto no role-playing (alguns role-playing são, a título demonstrativo e para minorar as ansiedades dos alunos, realizados pelos professores).

Os recursos materiais, além de uma sala apropriada para trabalho em grupo, são: aparelho para gravação de imagens, monitor, projetor.

\section{Cronograma, revisões e avaliações}

Além da avaliação permanente da atividade realizada em cada reunião, é feita avaliação sistematizada ao final de cada reunião e ao final da aplicação do projeto, permitindo uma reavaliação e reestruturação das atividades.

\section{Discussão}

Maguire et al. (1986) encontraram superioridade evidente nos profissionais treinados com a técnica de "vídeo-feedback interativa" frente à técnica convencional. Vários trabalhos posteriores têm confirmado a importância dessa técnica para o sucesso no ensino das habilidades de comunicação (Roter et al., 2004; Yedidia et al., 2003; Humphris, Kaney, 2000). Contudo, apesar das evidências favoráveis, poucos programas de treinamento para a entrevista médica utilizando esse formato têm sido implementados. Isto tem sido creditado, parcialmente, ao fato de esse formato ser considerado estressante, tanto por alunos como por professores, como aponta investigação realizada por Nielsen e Baerheim (2005). Nessa pesquisa, os autores identificaram, entre as principais preocupações: embaraço de ver sua atuação gravada, medo de demonstrar falta de conhecimento, medo de ser considerado inadequado em termos de personalidade ou em habilidades de comunicação, e medo de ser considerado incompetente.

A avaliação preliminar de nossa experiência tem confirmado tanto a eficácia da técnica quanto a necessidade de uma atenção especial aos fatores estressantes mobilizados. De um lado, verificamos que a filmagem de diferentes situações de entrevistas, protagonizadas tanto pelos alunos quanto pelos professores, podem facilitar significativamente a observação e treinamento nas técnicas de comunicação e de entrevistas. O exame cuidadoso e detalhado das imagens gravadas tem se revelado um instrumento poderoso para a detecção e evolução das capacidades relacionais e comunicacionais. Por outro lado, ficou patente a importância da manutenção de canais de escuta e avaliação permanentes, que permitam detectar a situação grupal e facilitar um direcionamento que evite a instalação e cristalização de defesas que possam obstaculizar o rendimento da atividade. Em relação ao estresse mobilizado pela atividade, por exemplo, temos buscado alternativas para minimizar esse inconveniente: os professores permanecem muito atentos a esse tópico, procurando respeitar 
e trabalhar as resistências. Em relação às gravações, uma fórmula que tem sido útil para minimizar o estresse nas turmas em que detectamos maiores resistências, é iniciá-las tendo como protagonistas os professores e, só depois, convidar os alunos a se envolverem na protagonização. Em relação ao feedback, que é outro fator de grande relevância para a produção de estresse, o cuidado que temos observado é em relação à criação de um clima grupal que favoreça a formulação de feedback construtivo, que seja descritivo e não valorativo, e que ressalte aspectos considerados funcionais, apontando, complementarmente, o que faltou ou o que poderia ser feito de outra forma, sugerindo e, eventualmente, protagonizando as sugestões. Para os alunos mais críticos (na maior parte das vezes, a crítica vem expressa em autocrítica), é sempre muito importante reassegurá-los de que o desempenho está adequado para o seu nível de experiência.

Os alunos, numa porcentagem significativa, manifestaram satisfação com a atividade, tanto nas avaliações informais como nas respostas a questionário (tipo Likert) aplicado ao final do semestre ( $81,6 \%$ dos alunos classificaram-na entre satisfatória e totalmente satisfatória, tendo em vista o objetivo de treinamento das habilidades e atitudes para a entrevista médica). Contudo, em relação à efetiva incorporação e manutenção das habilidades e atitudes, são necessárias avaliações mais sistematizadas, que já iniciamos e que serão estendidas no acompanhamento dos alunos ao longo do curso, tendo em vista rastrear a eficácia e repercussão deste preparo no desempenho dos estudantes em sua atividade na entrevista médica.

\section{Colaboradores}

Mario Alfredo De Marco coordenou e participou ativamente de todas as fases do trabalho (implantação do programa, pesquisa bibliográfica, redação do trabalho) e escrita final do manuscrito. Ana Luiza Vessoni trabalhou na implantação do programa, pesquisa bibliográfica e redação do trabalho. Augusto Capelo coordenou a gravação das imagens e participou ativamente de todas as fases; e Cíntia Camargo Dias coordenou o módulo e participou ativamente da implantação do programa, pesquisa bibliográfica e redação do trabalho.

\section{Referências}

ASPEGREN, K. Teaching and learning communication skills in medicine: a review with quality grading of articles. Med. Teach., v.21, p.563-70, 1999.

BARTLETT, E.E. et al. The effects of physician communications skills on patient satisfaction; recall, and adherence. J. Chronic Dis., n.37, p.755-64, 1984.

BORRELL, F. Entrevista clínica: manual de estratégias prácticas. Barcelona: SEMFYC, 2004.

CYRINO, E.G.; RIZZATO, A.B.P. Contribuição à mudança curricular na graduação da Faculdade de Medicina de Botucatu. Rev. Bras. Saude Mater. Infant., v.4, n.1, p.59-69, 2004.

DE MARCO, M.A. Do modelo biomédico ao modelo biopsicossocial: um projeto de educação permanente. Rev. Bras. Educ. Med., v.30, n.1, p.60-72, 2006.

Psicologia Médica. In: Casa do Psicólogo, 2003. p.77-80. (Org.). A face humana da Medicina. São Paulo: GARCIA, M.A.A. et al. A interdisciplinaridade necessária à Educação Médica. Rev. Bras. Educ. Med., v.31, n.2, p.147-55, 2007.

HENWOOD. P.G.; ALTMAIER, E.M. Evaluating the effectiveness of communication skills training: a review of research. Clin. Perform. Qual. Health Care, n.4, p.154-8, 1996. 
HULSMAN, R.L. et al. Teaching clinically experienced physicians communication skills: a review of evaluation studies. Med. Educ., v.33, n.9, p.655-68, 1999.

HUMPHRIS, G.M.; KANEY, S. The objective structured video exam for assessment of communication skills. Med. Educ., v.34, n.11, p.939-45, 2000.

MAGUIRE, P.; FAIRBAIRN, S.; FLETCHER, C. Consultation skills of young doctors: I-benefits of feedback training in interviewing as students persist. Br. Med. J. (Clin. Res. Ed.), v.292, n.6535, p.1573-6, 1986.

NILSEN, S.; BAERHEIM, A. Feedback on video recorded consultations in medical teaching: why students loathe and love it - a focus-group based qualitative study. BioMed Central Med. Educ., v.5, p.28, 2005.

RODRIGUEZ SALVADOR, J.J.; RUIZ MORAL, R. Programa de formación en técnicas de entrevista clínica: el proyecto COMCORD. Aten. Primaria, v.27, p.503-11, 2001.

ROSSI, O.S.; BATISTA, N.A. O ensino da comunicação na graduação em medicina - uma abordagem. Interface - Comunic., Saude, Educ., v.10, n.19, p.93-102, 2006.

ROTER, D.L. et al. Use of an innovative video feedback technique to enhance communication skills training. Med. Educ., v.38, n.2, p.145-57, 2004.

SANTOS, J.B. et al. Reflexões sobre o ensino de Semiologia Clínica. Rev. Bras. Educ. Med., v.27, n.2, p.147-52, 2003.

SAYD, J.D.; ANDRADE E SILVA, D.; RIBEIRO M.P.D. O aprendizado de Semiologia em um currículo tradicional. Rev. Bras. Educ. Med., v.27, n.2, p.104-13, 2003.

TRINDADE, E.M.V. et al. Resgatando a dimensão subjetiva e biopsicossocial da prática médica com estudantes de medicina: relato de caso. Rev. Bras. Educ. Med., v.29, n.1, p.48-50, 2005.

WISSOW, L.S.; KIMEL, M.B. Assessing provider-patient-parent communication in the pediatric emergency department. Ambulatory Pediatrics, v.2, n.4, p.323-9, 2002.

YEDIDIA, M.J. et al. Effect of communications training on medical student performance. JAMA, v.290, n.9, p.1157-65, 2003. 
O trabalho apresenta a atividade denominada "laboratório de comunicação" na qual, utilizando gravação e discussão de dramatizações de entrevistas (técnica de "vídeo feedback interativa") objetiva-se capacitar os estudantes de medicina nas habilidades comunicacionais necessárias para o exercício da tarefa médica. A atividade está inserida no segundo ano do curso médico, fazendo parte do programa de psicologia médica. O exame cuidadoso e detalhado das imagens registradas tem se revelado um instrumento muito poderoso para a detecção e a evolução das capacidades relacionais e comunicacionais. Ainda estamos em fase de realização de avaliações sistematizadas que nos permitirão verificar a eficácia da atividade e mensurar a repercussão que terá sobre o desempenho dos estudantes em sua atividade na prática médica.

Palavras-chave: Competência clínica. Comunicação. Relações médico-paciente. Educação médica.

\section{Communication laboratory: expanding medical students' skills for interview practice}

This paper presents an activity named "communication laboratory", in which, through recording and discussing simulated interviews ("interactive video feedback" technique), the aim is to capacitate medical students with the communicational skills needed for performing medical tasks. This activity is included in the second year of the medical course, and is part of the medical psychology program. Careful and detailed observation of the recorded images has revealed this to be a very powerful instrument for detecting and developing the relational and communicational capacities. We are still at the phase of performing systematized evaluations that will allow us, in the future, to verify the efficacy of the activity and to measure the repercussion that it will have on the development of students in their medical practice activities.

Keywords: Clinical competence. Communication. Physician-patient relations. Medical education.

\section{Laboratorio de comunicación: ampliando las habilidades del estudiante de medicina para la práctica de la entrevista}

El trabajo presenta la actividad denominada "laboratorio de comunicación", en la cual, utilizando grabación y discusión de dramatizaciones de entrevistas (técnica de vídeo feedback interactiva"), se trata de capacitar a los estudiantes de medicina en las habilidades de comunicabilidad necesarias al ejercicio de la tarea médica. La actividad se insiere en el segundo año del curso medico, formando parte del programa de psicología médica. El examen cuidadoso y detallado de las imágenes registradas se ha revelado instrumento muy poderoso para la detección y la evolución de las capacidades de relación y comunicación. Estamos aún en fase de realización de evaluaciones sistematizadas que nos permitirán comprobar la eficacia de la activad y medir la repercusión que tendrá sobre el desempeño de los estudiantes en su actividad en la práctica médica.

Palabras clave: Competencia clínica. Comunicación. Comunicación. Relación médicopaciente. Educación médica. 